\title{
Técnicas empleadas en síntesis de nanocompuestos en ingeniería de tejido óseo
}

\author{
Alis Yovana Pataquiva Mateus
}

Departamento de Ingeniería, Facultad de Ciencias Naturales e Ingeniería, Universidad Jorge Tadeo Lozano, Carrera 4 No. $22-61$ Bogotá, Colombia alisy.pataquivam@utadeo.edu.co

\section{Resumen}

Los avances en la ingeniería de tejido óseo exigen nuevos desarrollos en materiales inteligentes que permitan reemplazar hueso o bien regenerarlo. Para cumplir con esta tarea, la ingeniería biomédica, específicamente la ingeniería de los biomateriales, se ha basado en la nanotecnología ya que los tejidos son nanocompuestos naturales de perfecta arquitectura. El hueso, como nanocompuesto de colágeno y cerámico, es foco de bioinspiración a la hora de diseñar nuevos biomateriales para su tratamiento. Sin embargo, la complejidad de este tejido es enorme debido a las funciones críticas que desempeña tales como: movimiento y soporte de otros órganos vitales, almacenamiento de minerales, homeostasis, regulación del pH de la sangre y depósito de múltiples células tales como las mesenquimales, osteoblastos, condrocitos y fibroblastos, entre otras. De ahí, que es un reto para la ingeniería el diseño y la construcción de materiales con propiedades tales como biomimética, bioafinidad por las células óseas, así como cumplir con las propiedades mecánicas necesarias tales como resistencia a la compresión y a la tracción, entre otras condiciones mínimas. En esta revisión se hace alusión a algunas de las más novedosas técnicas en nanotecnología tales como nanopatrón, nanorecubrimiento y nanofibras, entre otras, empleadas en la construcción de diversos materiales nanoestructurados en el área de ingeniería de tejido óseo.

Palabras clave: biomateriales, ingeniería de tejidos, hueso, hidroxiapatita, células mesenquimales.

Editor: Hernández-Fernández J.

Citation: Pataquiva-Mateus A. (2014). Técnicas empleadas en síntesis de nanocomposites en ingeniería de tejido óseo. Revista Mutis 4(2).

Received: September 1, 2014; accepted: December 2, 2014; Published on line: December 31, 2014

Copyright: 02014 Pataquiva-Mateus A. This is an open-access article, which permits unrestricted use, distributions and reproduction in any medium, provided the original author and source are credited.

Competing Interests: The authors have no conflict of interest

\section{Used techniques in synthesis of nanocomposites in bone tissue engineering}

\begin{abstract}
Advances in bone tissue engineering require new developments in smart materials that allow replacement or regeneration of bone. To accomplish this task, biomedical engineering, specifically engineered biomaterials has been based on nanotechnology once the tissues are natural nanocomposites with perfect architecture. Bone, it is a nanocomposite based on collagen and ceramic, it is bioinspiration focus of the design to new biomaterials for treatment. However, its complexity is enormous tissue because it plays critical functions such as movement and support of other vital organs, storage mineral, homeostasis, regulation of blood $\mathrm{pH}$
\end{abstract}


and host of multiple cells such as mesenchymal cells and others. Hence, it is a challenge for engineering, designing and construction of materials with features such as biomimetics, bioaffinity by bone cells, as well as complying with the required mechanical properties, as minimum conditions. Some of the latest developments in nanotechnology used in the construction of several nanestructured materials in the area of bone tissue engineering are hinted in this overview.

Keywords: biomaterials, tissue engineering, bone, hydroxyapatite, mesenchymal stem cells.

\section{Introducción}

El hueso es considerado un nanocompuesto debido a que está conformado por una estructura blanda basada en colágeno, proteínas no colagenosas y agua; además de un componente duro inorgánico como es la hidroxiapatita $\left(\mathrm{Ca}_{10}\left(\mathrm{PO}_{4}\right)_{6}(\mathrm{OH})_{2}\right)$. De hecho, el $70 \%$ de la matriz ósea está compuesta por nanocristales de hidroxiapatita que usualmente tienen $20 \mathrm{~nm}$ de ancho y $80 \mathrm{~nm}$ de largo, aproximadamente. Otras proteínas que componen la matriz extracelular (ECM, de sus siglas en inglés) son también de dimensiones nanométricas, lo que proporciona un ambiente ideal para las actividades de adhesión, proliferación y diferenciación de células mesenquimales, osteoblastos, osteoclastos y fibroblastos (Engel, Michiardi, Navarro, Lacroix, \& Planell, 2007). Es por eso que la nanotecnología ayuda a entender cómo es la arquitectura de los tejidos y a diseñar las matrices (scaffolds) que serán colonizadas por las células propias de cada tejido antes o después de la implantación; de ahí que la ciencia de los biomateriales se enfoque en el diseño y la construcción de matrices apropiadas para el reemplazo o la regeneración del tejido, mediante herramientas que ofrece la nanotecnología tanto para la manipulación como para la caracterización de los materiales. En este artículo se revisan las tendencias de los nanocompuestos usados en la ingeniería de tejidos, específicamente el óseo.

\section{Nanotecnología aplicada en la ingeniería de tejido óseo}

La nanotecnología ha permitido el desarrollo de nuevos materiales con propiedades mejoradas tales como eléctricas, ópticas, catalíticas, magnéticas e incluso mecánicas y bioactivas; siendo estas últimas de gran interés en la ingeniería de tejido óseo. Los nanomateriales incluyen nanopartículas, nanoclusters, nanocristales, nanotubos, nanofibras, nanocables, nanobarras, nanopelículas, y nanorecubrimientos, entre otros, que son obtenidos mediante las dos técnicas para sintetizar estos materiales a escala nanométrica que son: top-down y bottom-up; dentro de las que se cuentan electrospinning, separación de fases, sol-gel, procesos de autoensamblado, deposición de películas, deposición química de vapor (CVD, de sus siglas en inglés), grabado químico, nanoimpresión, fotolitografía, etc. Estas técnicas facilitadas por la nanotecnología son capaces de procesar nanomateriales con topografías nanométricas tanto al azar como ordenadas y crecimiento o autoensamblado de los materiales que simulan las dimensiones de sus contrapartes naturales, con un aumento dramático en el área superficial, rugosidad, humectabilidad y proporciones área/ volumen que mejoran notablemente su actividad biológica in vitro e in vivo, siendo la osteointegración y osteoconducción las propiedades más deseadas para un biomaterial con fines de regeneración ósea.

Los problemas relacionados con el tejido óseo (fracturas, osteoartritis, osteoporosis y cáncer) siempre han representado significativos problemas en el sistema de salud, sin contar con los tiempo de recuperación, dolor, tiempos de licencias médicas, etc. De hecho, el National Center for Health Statistics (NCHS) reportó más de un millón de fracturas en Estados Unidos para el año 2004 y la Academia Americana de Cirujanos Ortopédicos reportó que en un período de 4 años se había aumentado en más de un $80 \%$ los casos de reemplazo de cadera para ese mismo año (AAOS, 2004). A nivel mundial se prevé que para año 2025 ocurran 2.6 millones de fractura de cadera, cifra que ascendería casi al doble en el 2050 (Sterling, 2011).

\section{Nanopatrones}

La topografía de los nanomateriales se ha convertido en un foco de numerosos estudios debido a que las características físicas de la superficie son de vital importancia por su estrecha relación con la actividad celular; por lo cual, se han desarrollado varias técnicas para modificar de manera oganizada o aleatoria la superficie de nanomateriales, entre las cuales se destacan: litografía coloidal (colloidal lithography), estampado en caliente (hot embossing), litografía por haz de electrones o iones (e-beam/ion-beam lithography), litografía de inmersión de punta (dip-pen li- 
thography), demezclado de polímeros (polymer demixing), grabado químico (chemical etching), etc. (Engel, Michiardi, Navarro, Lacroix, \& Planell, 2007).

En el caso específico de regeneración de tejido óseo, existe una necesidad de desarrollar nuevos biomateriales implantables que biomimeticen la matriz ósea ya que el hueso es un compuesto nanoestructurado. Recientemente se ha desarrollado un biochip que tiene ranuras grabadas en su superficie para el contacto con osteoblastos, con el objetivo de tratar enfermedades óseas tales como osteoporosis y artrosis. Se encontró que los osteoblastos mostraron mineralización a nanopatrones menores a $75 \mathrm{~nm}$ y $33 \mathrm{~nm}$ de ancho y profundidad, respectivamente (Lamers, y otros, 2010).

\section{Nanorecubrimientos}

Nanomateriales de fase nanométrica también pueden ser depositados en la superficie de un biomaterial con miras a mejorar su bioactividad o biocompatibilidad. De hecho, son bien conocidos los recubrimientos con hidroxiapatita nanocristalina mediante el método sol-gel o bien por electrodeposición de pulso (Saremi \& Golshan, 2007) (Caruso \& Antonietti, 2001), las cuales se usan para mejorar la osteointegración de un dispositivo ortopédico.

Los biomateriales poliméricos vienen siendo utilizados ampliamente en reparación y regeneración ósea debido a sus excelentes propiedades mecánicas, sin embargo poseen una pobre osteointegración como una baja angiogénesis por lo que partículas inorgánicas bioactivas pueden servir como recubrimiento de estos biomateriales para mejorar estas propiedades in vitro e in vivo mediante una sola técnica. Para lograr este objetivo se han realizado estudios (Wu, y otros, 2014) empleando la técnica de deposición por láser pulsado (PLD, de sus siglas en inglés) para preparar una nanocapa uniforme de akermanita $\left(\mathrm{Ca}_{2} \mathrm{MgSi}_{2} \mathrm{O}_{7}, \mathrm{AKT}\right)$ sobre polisulfona (PSU) y ácido poliláctico (PLA). Los resultados exitosos se explican debido a que la AKT es una cerámica con contenido de $\mathrm{Si}, \mathrm{Ca}$ y $\mathrm{Mg}$ haciéndola bioactiva debido a que se promueven la diferenciación de las células responsables por la formación de hueso y de los vasos sanguíneos a partir de células estromales de médula ósea. Físicamente, el recubrimiento con AKT mejoró propiedades tales como: hidrofilicidad, rugosidad, dureza y habilidad de mineralizar apatita comparadas con los sustratos (PSU y PLA) desnudos.

\section{Nanofibras}

El electrospinning es un método muy empleado en la fabricación de micro/nanofibras. En este proceso una solución polimérica puede ser expulsada desde una jeringa y se conforma en una gota suspendida en la punta de la boquilla. Cuando un campo eléctrico se aplica a la punta de la boquilla, la fuerza electrostática que actúa sobre la solución polimérica suspendida supera la tensión superficial de la solución, se generan fibras a escala micro o nanométrica. Con el uso de este tipo de técnica se fabricaron materiales micro y nanofibrosos de policaprolactona ( $\mathrm{PCL}$ ) con varios contenidos de phlorotanning que es un extracto algal que contiene varios factores de crecimiento que promueven la regeneración de varios tejidos (Kim \& Kim, 2012); encontrándose una hidrofilicidad marcada y propiedades mecánicas mejoradas nueve veces que las encontradas en el PCL puro. La presencia de phlorotanning mostró mayores viabilidad celular y mineralización, haciendo de la dupla material micro/nanofibroso y un factor de crecimiento natural, un prometedor biomaterial en la regeneración de tejido óseo.

\section{Nanopartículas y nanotubos}

La hidroxiapatita es la molécula base para muchos de los biomateriales óseos debido a que este fosfato de calcio es el componente inorgánico de la matriz del hueso. Muchos son los métodos para la síntesis de hidroxiapatita destacándose la ruta de precipitación química que ha sido ampliamente empleada debido a su facilidad y bajo costo. La precipitación química se caracteriza por la presencia de una fuente de fósforo como el ácido ortofosfórico y una fuente de calcio como el hidróxido de calcio, que reaccionan bajo condiciones controladas de operación (condiciones de las materias primas, $\mathrm{pH}$, temperatura, agitación, velocidad de adición del ácido, dimensiones del goteamiento, etc.). Es importante resaltar que las concentraciones de los reactivos influyen en la clase de especie o especies producidas en la reacción, así como la participación de surfactantes que ayudan a mantener lo más pequeños posibles los cristales de fosfatos de calcio formados, asegurando en gran medida la obtención de nanopartículas (Pataquiva-Mateus, Barrias, Ribeiro, Ferraz, \& Monteiro, 2007). En la figura 1 se aprecia la morfología del material sintetizado por esta ruta empleando concentraciones estequiométricas, mientras que en la figura 2 se verifican los picos característicos correspondientes al grupo fosfato en 
las bandas $560-633 \mathrm{~cm}^{-1}$ y cerca de $1030 \mathrm{~cm}^{-1}$. El uso de surfactantes tales como SDS (dodecilsulfato de sodio) permite la formación de hidroxiapatita sintética y b-TCP el cual es un fosfato de calcio de alta biodegradabilidad; mezcla de atractivo biomédico por sus propiedades mecánicas, biocompatibles y biodegradables nanopartículas (Pataquiva-Mateus, Barrias, Ribeiro, Ferraz, \& Monteiro, 2007).

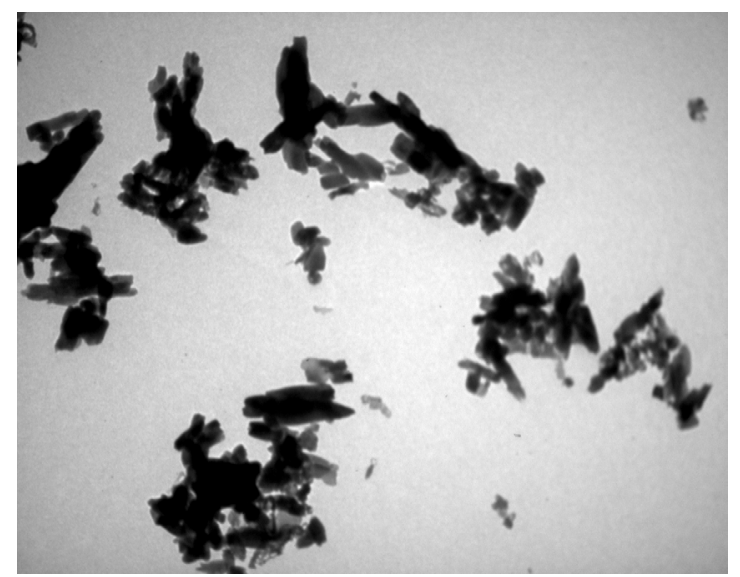

Figura 1. Micrografías de transmisión electrónica de hidroxiapatita sintética en polvo $(320,000 X)$

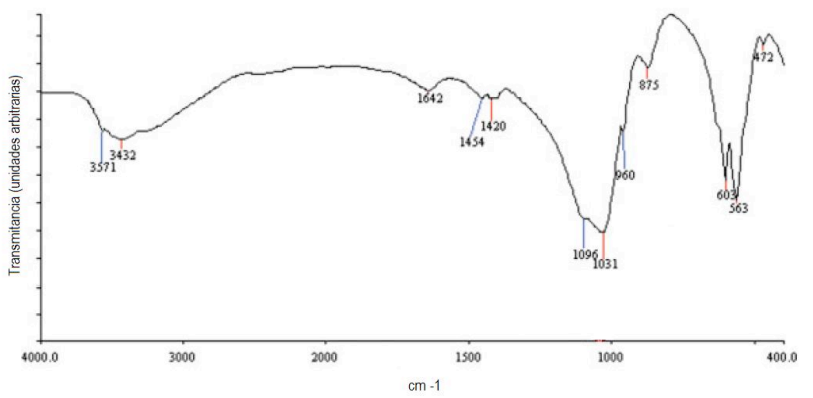

Figura 2. Espectro de Fourier Transform Infrared (FTIR) de hidroxiapatita en polvo por método de precipitación química

El método sol-gel es un proceso que se lleva a cabo a baja temperatura para la obtención de vidrios bioactivos mediante la hidrólisis y policondensación de hidróxidos metálicos, alcóxidos y sales inorgánicas; encontrándose mejores propiedades de adhesión ósea, mayores velocidades de degradación y habilidad de incorporar varios cationes, haciendo de esta ruta de síntesis de nanopartículas una de las más empleadas y ampliamente difundidas como es el método de precipitación química (Arcos \& Vallet-Regi, 2010; Jones, 2010). Variables de proceso tales como el pH, concentración de los precursores y temperatura de síntesis, han demostrado modificar la red de sílice afectando la estructura final del vidrio. De hecho el uso de catalizadores básicos han permitido la obtención exitosa de nanopartículas de vidrio bioactivo en el rango de 30$100 \mathrm{~nm}$ a través de esta técnica conjugada con el método de coprecipitación. Investigaciones empleando la técnica de sol-gel demostraron la capacidad de soportar osteoblastos humanos y permitir su proliferación y diferenciación (Fan, Kaila, Di Silvio, \& Huan, 2014).

Por otro lado, los nanotubos se presentan como un material alternativo de interesantes propiedades a nivel de actividad biológica tales como: flexibilidad y nanoestructura que promueve la deposición de hueso en la superficie (Popat, Leoni, Grimes, \& Desai, 2007). De hecho estudios realizados (Gao, y otros, 2014) mostraron que superficies nanoestructuradas a partir de nanotubos de carbono de dióxido de titanio fueron estudiados en cuanto a su viabilidad celular, demostrando que estas superficies soportaron alta adhesión celular, proliferación y viabilidad hasta por una semana de cultivo, cuando se compararon con superficies de titanio.

\section{Microdispositivos que emplean nanomateriales}

En los últimos años ha aumentado el interés de usar la experiencia y el conocimiento que se tiene de los sistemas miomicroelectrónicos (BioMEMS) dentro de los dispositivos a escala nanométrica y que han sido posibles gracias a la tecnología de microfabricación permitiendo obtener detectores o actuadores a nanoescala (Bashir, 2004) y específicamente en biomedicina (Khademhosseini, Bettinger, Karp, Ling, \& Borenstein, 2006) donde se destacan biocápsulas, biorreactores, biosensores y labs-on-a-chip. Las primeras son nanodispositivos que permiten almacenar, transportar y liberar controladamente moléculas tales como fármacos, proteínas, enzimas, etc. De hecho, aislar moléculas puede ser viable en este tipo de dispositivos una vez que es posible diseñar la biocápsula para que deje almacenar moléculas menores a $6 \mathrm{~nm}$ y excluir las mayores a $15 \mathrm{~nm}$ (Desai, 2000). Como es de esperarse, el impacto que tendrían estas biocápsulas en la medicina regenerativa es enorme, debido a su amplio abanico de posibilidades entre las que se destacaría el uso como herramienta de diagnóstico local en tiempo real para la prevención o tratamiento de enfermedades, cuando es encapsulado un nanodispositivo combinado con un sistema de liberación controlada.

Los biorreactores por su parte, son dispositivos donde las células regenerativas y tejidos son cultivados bajo 
condiciones controladas $(\mathrm{pH}$, temperatura, presión, flujo de nutrientes, etc.) de operación y monitoreo abriendo la posibilidad de llegar a aplicaciones a escala industrial, gracias al acoplamiento de diversos tipos de BioMEMS. Así mismo, los biosensores y los lab-on-achip son integrados a los biorreactores para monitorear y detectar respuestas celulares específicas, que aunque son producto de la microtecnología, la aplicación a escala nanométrica promete grandes avances en las aplicaciones de bionanosensores, los cuales reaccionarían a ambientes locales mediante respuestas ópticas o eléctricas. De hecho se espera que nanosensores sean capaces de regular automáticamente un cultivo por ciclos de retroalimentación mejorando considerablemente la regeneración de tejidos. Como ejemplos se cuentan los puntos cuánticos, nanopartículas fluorescentes, y nanopartículas metálicas, entre otros (Engel, Michiardi, Navarro, Lacroix, \& Planell, 2007).

\section{Conclusiones y perspectivas futuras}

La perspectiva de reemplazar tejido damnificado por uno regenerado cambiaría definitivamente la ciencia médica tanto desde el punto de vista médico como social. El interés de usar nanotecnología en medicina regenerativa es creciente, debido a su capacidad de producir estructuras a escala nanométrica capaces de remedar los tejidos naturales así como las nanopartículas para el uso de entrega controlada de macromoléculas. En la actualidad son varias los frentes de trabajo alrededor del tema de ingeniería de tejidos incluyendo el óseo; uno de ellos es el trabajo en biorreactores donde el biomaterial diseñado es puesto en contacto con factores de crecimiento y células sanas provenientes del paciente para su posterior implantación directa. Otro frente de trabajo es el de desarrollar biomateriales que, valiéndose de sus propiedades, son capaces de enviar las señales físicas y químicas para que las células usen el material como epicentro de sus actividades biológicas mientras se va produciendo la regeneración del tejido. A este tipo de biomateriales usualmente se les consideran smart materials debido a que, aun sin poseer células en su interior, podrían iniciar el proceso de regeneración debido a sus características físicas y propiedades mecánicas. Trabajos paralelos se han desarrollado en cuanto a la influencia celular, mediante la señalización de moléculas inmovilizadas donde el desempeño celular es controlado mediante dosis y distribución de sustancias bioactivas, incluidas en estos biomateriales inteligentes, lo que hace de este tema un foco de investigaciones emergentes a nivel mundial en la ingeniería de tejidos.

\section{Referencias}

AAOS, A. A. (2004). http://www.aaos.org/Research/ stats/patientstats.asp.

Arcos, D., \& Vallet-Regi, M. (2010). Sol-gel silica-based biomaterials and bone tissue regeneration. Acta Biomaterialia, 6, 2874-2888.

Bashir, R. (2004). State-of-the-art in detection, opportunities and prospects. Advanced Drug Delivery Reviews, 56, 1565-1586.

Caruso, R., \& Antonietti, M. (2001). Sol-gel nanocoating: an approach to the preparation of structure materials. Chemistry of materials.

Desai, T. (2000). Micro- and nanoscale structures for tissue engineering constructs. Medical Engineering \& Physics, 22, 595-606.

Engel, E., Michiardi, A., Navarro, M., Lacroix, D., \& Planell, J. (26 de noviembre de 2007). Nanotechnology in regenerative medicine: the materials side. Cell, 26(1), 39-47.

Fan, J., Kaila, P., Di Silvio, L., \& Huan, J. (2014). In vitro response of human osteoblasts to multi-step solgel derived bioactive glassnanoparticles for bone tissue engineering. Materials Science and Engineering $C$, 206-214.

Gao, A., Hang, R., Huang, X., Zhao, L., Zhang, X., Wang, L., . . Chu, P. (2014). The effects of titania nanotubes with embedded silver oxide nanoparticles on bacteria and osteoblasts. Biomaterials, 35, 4223-4235.

Jones, J. (2010). Review of bioactive glass: from Hench to hybrids. Acta Biomaterialia, 9, 4457-4486.

Khademhosseini, A., Bettinger, C., Karp, J. Y., Ling, Y., \& Borenstein, J. (2006). Interplay of biomaterials and microscale technologies for advancing biomedical applications. Journal of Biomaterials Science Polymer Edition, 17, 1221-1240.

Kim, M., \& Kim, G. (2012). Electrospun PCL/ phlorotanning nanofibres for tissue engineering: Physi- 
cal properties and cellular activities. Carbohydrate Polymers, 592-601.

Lamers, E., Walbooners, X., Domanski, M., Riet, J., van Delft, C., Luttge, R., . . . Jansen, J. (2010). The influence of nanoscale grooved substrates on osteoblast behaviour and extracellular matrix deposition. Biomaterials, 3307-3316.

Pataquiva-Mateus, A., Barrias, C., Ribeiro, C., Ferraz, M., \& Monteiro, F. (2007). Comparative study of nanohydroxyapatite microspheres for medical applications. Journal of Biomedical Materials Research Part A, 86A, 483-493.

Popat, K., Leoni, L., Grimes, C., \& Desai, T. (2007). Influence of engineered titania nanotubular surfac- es on bone cells. Biomaterials, 28, 3188-3197.

Saremi, M., \& Golshan, B. (2007). Microstructural study of nano hydroxyapatite coating obtained by pulse electrodeposition process on TI-6AI-V-4. Transactions of the Institute of Metal Finishing, 99-102.

Sterling, R. S. (2011). Gender and race/ethnicity differences in hip fracture incidence, morbidity, mortality, and function. Clinical Orthopaedics and Related Research ${ }^{\circledast}, 469(7), 1913-1918$.

Wu, C., Zhai, D., Ma, H., Li, X., Zhang, Y., Zhou, Y., . . . Chang, J. (2014). Stimulation of osteogenic and angiogenic ability of cells on polymers by pulsed laser deposition of uniform akermatinte-glass nanolayer. Acta Biomaterialia. 